been measured and discussed, a few preliminary statements and indications may be of value.

(I) Foremost among these is one of disappointment in that no faint stars with very large motions have been found; not a single new motion exceeding 3 " annually, only 3 new stars with motions between $2^{\prime \prime}$ and $3^{\prime \prime}$ per annum, and no stars of the I 7 th magnitude or fainter with a motion as large as $I^{\prime \prime}$ annually.

In the supposedly much better known northern hemisphere Ross found, among some 800 new motions, one star with a motion as large as $5^{\prime \prime}$ and one other of more than $3^{\prime \prime} \cdot 5$ while recently a third, with a motion of $3^{\prime \prime} \cdot 7$ annually, was found at Minnesota, also in the northern hemisphere. While it is possible that the southern hemisphere is unusually poor in large proper motion stars, the present result does tend to indicate that stars of extremely low luminosity are not as abundant in space as has been claimed by recent investigators.

2. There appear to be some indications of a non-uniform distribution of proper motion stars over the sky, even after allowance is made for proximity to the apices of the various drifts.

3. A preliminary discussion of the favoured directions of motion-star streaming -is in progress, and promises to yield the usual results.

4. While more than Iooo double stars-wide pairs with common proper motion - have been found, not a single bona-fide triple and certainly no conspicuous moving clusters have been observed.

5. No faint distant companions to bright stars of large proper motion have been found, even though a special lookout was kept for such stars.

6. Some I200 new variable stars have been found, mainly belonging to the long-period class. Not a single certain variable with proper motion has been found, and only two cases where either the variability or the proper motion is only suspected, which result will have some bearing on the frequency of eclipsing binaries and W Ursae Majoris stars among dwarfs of low luminosity.

7. No proper motions of the Nova Pictoris type have been found, i.e. no stars apparently running away in opposite directions from the scene of an explosion. While it would be too much to say that such objects had been expected, we must not overlook the fact that, e.g., the B and C components of Nova Pictoris itself will probably be a matter of $3^{\prime}$ apart in rooo years, and by that time nothing except a record of their history would indicate a former association.

Dr Nechvile reports that the region with centre at R.A. $6^{\mathrm{h}} 30^{\mathrm{m}}$, Decl. $+24^{\circ}$ has been examined in collaboration with $M$. Bosler of the Marseille Observatory.

\title{
COMMISSION 25. (STELLAR PHOTOMETRY.)
}

\author{
Acting President: Prof. Ő. Bergstrand. \\ Secretary: Prof. B. Lindblad.
}

The acting chairman read a letter from the President of the Commission, $\mathrm{Mr}$ F. H. Seares, calling the attention of the Commission to certain points of importance which might be taken up for discussion. In the course of this discussion $\mathrm{Dr} H$. Shapley invited cooperation with the Harvard Observatory on the work of unification of the magnitude systems in the northern and southern sky.

After a discussion on the problems of standardizing colour filters and photographic plates used in stellar photometry the recommendation suggested by Dr Shapley 
that all engaged in photometric research publish promptly the characteristics of their instruments, colour filters, etc., was adopted.

The report presented by the President of the Commission was accepted without alteration.

\section{COMMISSION 26. (DOUBLE STARS.)}

President: Prof. E. Hertzsprung.

SeCRETARY: Dr W. H. VAN DEN Bos.

The President read the draft report. No objections being entered, this was adopted by the meeting.

The President proposed an addition to the report. This was agreed to.

The adoption of a single system of orbital elements was advocated by van den Bos, who reported on the correspondence which had taken place on this subject with the members. As the result of all answers received, a system of elements was proposed and adopted by the Committee.

The President suggested that this be given in the report and van den Bos, at the request of van Biesbroeck, proposed that a full set of definitions and formulae be added. This was agreed to.

Salet asked if Committee 3 (notations) should not be consulted: van den Bos replied that all the symbols in question are already in general use and that his proposal is not a matter of notation, but of unique definition of the existing symbols.

Zagar reported for Bianchi that the $50-\mathrm{cm}$. refractor of Milan will be re-erected at Merate, to be used for visual measures in connection with photographic measures with the roo-cm. reflector.

Zagar expressed the wish for more radial velocities of visual doubles and for publication of the times of observation.

The President replied that this can be better achieved by personal correspondence.

Giacobini proposed an international distribution of the pairs to be measured over observers, measurement of neighbouring stars for deriving mass ratios and the establishment of a list of personal equation stars.

The President replied that the first two proposals are of too wide a scope to be discussed at this meeting and that for the third Giacobini's own list might be considered.

Arend reported that Uccle Observatory plans the remeasurement of the pairs observed there by van Biesbroeck in earlier years, with new optical equipment.

Commission 26 requests computers of binary star orbits to publish in future the following data, using the symbols only in the sense indicated here:

\section{Elements}

$P$ Period. In years unless otherwise stated.

$n$ Mean motion, $=360 \%$. Always positive.

$T$ Epoch of periastron passage. Not to be corrected for light-time.

e Eccentricity.

a Semi axis major of relative orbit, in seconds of arc. 